\title{
Germanica
}

\section{L'impossible généalogie féminine chez Ingeborg Bachmann}

Von der Unmöglichkeit einer weiblichen Genealogie bei Ingeborg Bachmann

Herta Luise Ott

\section{CpenEdition}

\section{Journals}

Édition électronique

URL : http://journals.openedition.org/germanica/1327

DOI : 10.4000/germanica.1327

ISSN : 2107-0784

\section{Éditeur}

Université de Lille

Édition imprimée

Date de publication : 30 juin 1998

Pagination : 123-134

ISBN : 9770984263203-22

ISSN : 0984-2632

\section{Référence électronique}

Herta Luise Ott, «L'impossible généalogie féminine chez Ingeborg Bachmann », Germanica [En ligne],

22 | 1998, mis en ligne le 31 janvier 2012, consulté le 06 octobre 2020. URL : http://

journals.openedition.org/germanica/1327 ; DOI : https://doi.org/10.4000/germanica.1327

Ce document a été généré automatiquement le 6 octobre 2020.

(c) Tous droits réservés 


\title{
L’impossible généalogie féminine chez Ingeborg Bachmann
}

Von der Unmöglichkeit einer weiblichen Genealogie bei Ingeborg Bachmann

\author{
Herta Luise Ott
}

1 La prose tardive d'Ingeborg Bachmann se lit comme une perpétuelle mise en scène d'un conflit mortel entre hommes et femmes, qui laisse les femmes à l'état de victimes. Présenté d'une manière assez crue dans certains fragments du cycle de romans Todesarten ${ }^{1}$,ce conflit apparaît sous forme «travaillée » dans Malina, le seul roman que Bachmann ait publié de son vivant ${ }^{2}$.

2 Dans ses œuvres antérieures, hommes et femmes vivent, au niveau de la narration, des conflits certes moins sanguinaires, mais ils se voient fréquemment opposés selon le paradigme suivant : la femme se voue entièrement à l'amour d'un homme, l'homme se laisse temporairement séduire par cet être étrange pour ensuite retourner dans un monde « réel ». Le personnage emblématique de cette constellation est la sirène Ondine du récit Undine geht $(\mathrm{W} 2)^{3}$. Pourtant, le personnage féminin central semble avoir ici la fonction de représenter l'amour et l'art en général de manière allégorique plutôt que de mettre en scène une femme « réelle $»^{4}$.

3 Bachmann choisit fréquemment, à cette époque, une perspective narrative «masculine» ou neutre, dès qu'il n'est plus exclusivement question de l'amour à la Ondine : dans ces récits, la voix narrative accompagne un personnage masculin, voire elle se manifeste par sa bouche. On peut alors caractériser comme fonctionnelle la manière dont Bachmann emploie les voix narratives. Le discours raisonné, le questionnement sur les échecs de la raison et les limites de l'expérience appartiennent aux hommes, mais la déraison, la mise en question radicale de cette raison, de l'ordre du monde, se font entendre à travers la bouche d'une femme. Il y a donc en quelque sorte polyphonie "raisonnée " des voix narratives dans un monde « masculin", ceci aussi bien dans la prose que dans les pièces radiophoniques. Ce qui intéresse Bachmann alors, ce n'est pas la question des relations entre hommes et femmes, mais plutôt l'appréhension et la représentation d'un monde où la loi de la société barre toute 
aspiration vers un absolu de l'expérience, que celui-ci soit d'ordre poétique ou pratique.

4 Par la suite, on peut observer un changement radical dans l'écriture de Bachmann. Nous assistons d'abord, dans le discours Ein Ort für Zufälle (W4), prononcé à l'occasion de la remise du prix Georg Büchner en 1964, à une désagrégation du sujet de la narration ${ }^{5}$. L'unité du texte et des images garantit certes une unité de la perspective narrative, mais la plupart du temps il est impossible de cerner un narrateur individualisé : s'agit-il d'un(e) malade ou d'un collectif de malades ?

5 À cette époque, Bachmann travaille déjà sur un projet "Todesarten » (cf. TP 1,489). Nous voyons se dessiner, autour du fragment Franza, deux problématiques : d'une part, il est question d'un amour mortel qu'une femme porte à un homme qui s'avère être son bourreau. Elle éprouve en même temps un amour de caractère incestueux, non consommé, pour son frère ${ }^{6}$. D'autre part, Bachmann expérimente longtemps les voix narratives en oscillant entre celle du frère, de la sœur et une voix neutre (cf. TP2). Finalement, elle remettra à plus tard ce projet pour écrire Malina.

Ce roman, publié en 1971, est conçu comme « ouverture » d'un cycle de romans dont le titre prévu était Todesarten; il représente en quelque sorte une somme de la prose d'Ingeborg Bachmann ${ }^{7}$. Ce qui est mis en scène ici, ce n'est plus le discours d'une voix féminine qui exprime le désir d'un amour, d'une expérience absolue, mais la mort du sujet parlant féminin qui aspire en vain à une réalisation de ses désirs érotiques. Le MOI féminin parle, certes, mais il le fait pour constater sa propre destruction. Cet article se propose d'analyser le fonctionnement de cette puissance (masculine ?) à laquelle le MoI féminin ne semble rien pouvoir opposer. Il convient donc de cerner les images et les structures narratives qui peuvent nous guider vers celle-ci et d'en chercher l'impact sur d'autres textes que Bachmann a écrits par la suite.

7 Dans l'introduction du roman, il est question d'une première rencontre décisive que fait la narratrice avec le sexe opposé, en dehors de la famille. Nous pouvons lire à ce propos :

Am Anfang der Seepromenade des Wörthersees, nicht weit von der Dampferstation, bin ich zum ersten mal geküßt worden, aber ich sehe kein Gesicht mehr, das sich meinem nähert. (W3, 24)

Mais cette rencontre est aussitôt relativisée :

... nur war ich damals schon neunzehn Jahre alt und nicht mehr sechs, mit einer Schultasche auf dem Rücken, als es wirklich passierte. In einer Großaufnahme steht die kleine Glanbrücke da, nicht das abendliche Seeufer, nur diese mittäglich übersonnte Brücke mit den zwei Buben, die auch ihre Schultasche auf dem Rücken hatten... [...] Es war auf der Glanbrücke. Es war nicht die Seepromenade (W3, 24 sq).

9 Selon une longue tradition littéraire, les traversées de fleuves évoquent métaphoriquement un passage, une coupure importante dans la vie des protagonistes. Ingeborg Bachmann la décrit, pourrait-on dire, comme une photographie imaginaire. Moi parle d'un "gros plan », qui se substitue à un autre souvenir ainsi annulé(celui du premier baiser). Mais cette image évoque aussitôt un troisième souvenir, annulé par la suite, lui aussi : une traversée de New York vers l'Europe, pendant laquelle a lieu son anniversaire. Pourtant, cette expérience ne paraît pas avoir, elle non plus, une valeur décisive :

Es war nicht auf der Glanbrücke, nicht auf der Seepromenade, es war auch nicht auf dem Atlantik in der Nacht. Ich fuhr nur durch diese Nacht, betrunken, der untersten Nacht entgegen. (W3,26) 
Ce souvenir la fait penser à un film sur Venise, " das ich nie sehen werden » (W3, $26 \mathrm{sq)}$ film qu'elle a vu il y a longtemps et dont elle garde en mémoire une musique qu'elle n'a plus jamais entendue sans être dérangée (W3, 26 sq). Finalement, MOI abandonne cette trame du récit, car « es stört mich alles in der Erinnerung » (W3, 27). Elle n'arrive donc pas, contrairement à ce qu'on pourrait attendre après ces longs préliminaires, à fixer une «image originelle ». Tout ce qui lui reste finalement, c'est la mémoire d'une mélodie qui s'évanouit dans des cris.

11 Le roman fait référence par la suite à des souvenirs bien différents, mais ces images aquatiques gardent une fonction importante dans l'économie du récit. Elles renvoient, entre autres, à une scène-clé dans le chapitre des rêves : celle où MoI comprend enfin que son père est «zum Theater gegangen », que Dieu est « eine Vorstellung » (W3, 180-181). La loi que représente le père et à laquelle MoI est soumise se présente donc ici comme purement imaginaire. Ce qui n'évite pas que MoI arrive, vers la fin du chapitre des rêves « in der untersten Nacht » $(\mathrm{W} 3,235)$, là où son père porte des costumes de bourreau, tachés de sang, horribles, dont il change sans cesse, ressemblant ainsi à cette « image originelle » toujours renouvelée.

Mais revenons à l'histoire qu'a vécue MoI sur le fleuve :

... der ältere, mindestens zwei Jahre älter als ich, rief : Du, du da, komm her, ich geb dir etwas! Die Worte sind nicht vergessen, auch nicht das Bubengesicht, der wichtige erste Anruf, nicht meine erste wilde Freude, das Stehenbleiben, Zögern, und auf dieser Brücke der erste Schritt auf einen anderen zu, und gleich darauf das Klatschen einer harten Hand im Gesicht: Da, du, jetzt hast du es! Es war der erste Schlag in mein Gesicht und das erste Bewusstsein von der tiefen Befriedigung eines anderen, zu schlagen. Die erste Erkenntnis des Schmerzes (W3, 24).

13 La rencontre avec le sexe opposé, d'abord projetée comme rencontre amoureuse (le premier baiser) aboutit ici à une scène dominée par la violence gratuite qu'exercent des garçons, des mâles, sur la petite fille.

Ingeborg Bachmann entend créer dans ce roman une base qui lui serve de référence pour un livre à venir ${ }^{8}$. Quelle en est la nature?

Bien que l'image soit annulée par la suite, la rencontre sur le pont implique de lourdes conséquences : la fille, n'ayant pas réussi à franchir la frontière qui la sépare du monde extérieur (elle revient sur ses pas après avoir été battue), restera prisonnière de la constellation familiale. Il y aura répercussion de cet emprisonnement tout au long du roman. La constellation familiale, centrée ensuite sur la relation fille-père, joue un rôle primordial même dans un espace social apparemment "neutre ». Face à une figure paternelle, la femme a du mal à définir sa place par rapport aux autres membres de la famille, en particulier la mère. Ainsi, MoI essaie de s'affirmer vis-à-vis des enfants de son bien-aimé Ivan, mais elle refuse le rôle de l'adulte, celui d'une mère.

Le phénomène ne se limite d'ailleurs pas au roman Malina. Dans le récit Drei Wege zum See, paru environ un an et demi plus tard, en 1972, Elisabeth Matrei, le personnage principal, revendique le rôle de mère face au frère, rivalisant ainsi avec sa propre mère ${ }^{9}$. Mais elle porte aussi le même nom que la mère de son amant Franz Joseph Eugen Trotta, ce qui ne relève pas du hasard chez Ingeborg Bachmann.

17 Dans le fragment narratif Gier, publié après sa mort ${ }^{10}$, le veuf Bertolt Rapatz emploie Elisabeth Mihailovics comme bonne d'enfants pour ensuite l'épouser. Mais elle se sent trop jeune pour occuper un rôle de mère auprès de sa belle-fille. 

occupent à l'intérieur d'une structure familiale ou sociale. Leurs relations avec d'autres membres de la famille leur semblent incorrectes ou "fausses" et sur un plan imaginaire, elles sont incapables de déterminer leur place en tant que femmes vis-à-vis d'autres femmes. Aucune d'elles ne semble vouloir ou pouvoir occuper la place devenue vacante de la mère, mais en même temps elles se trouvent toutes en concurrence par rapport à une mère - attitude qui se manifeste surtout chez Elisabeth Matrei ${ }^{11}$. - Ayant nié la position de la mère, les femmes n'arrivent ni à se démarquer d'elle ni à occuper sa place. Voilà comment est levée la barrière entre les générations. MoI se perçoit elle-même, ainsi que sa mère et sa sœur, comme occupant des positions interchangeables face au père en tant qu'objets de ses appétits sexuels (par ex. W3, 190 et 212). C'est lui qui détient le pouvoir sexuel et il en profite pleinement. La mère, qui a perdu sa place de partenaire du père, ne semble pas pouvoir s'opposer à cet homme tout-puissant qui, lui, finit même par occuper la place de la mère face à la fille : « Mein Vater hat diesmal auch das Gesicht meiner Mutter, ich weiß nie genau, wann er mein Vater und wann er meine Mutter ist... » (W3,233)

Derrière le masque du père apparaît donc dans Malina une figure non spécifiée sexuellement. Cette figure indéfinissable et toute-puissante d'assassin représente-t-elle alors un pouvoir paternel, maternel ou neutre, au-delà de toute attribution sexuelle ? Il est difficile, sinon impossible d'y répondre. À partir d'une analyse de Drei Wege zum See, tout ce que nous pouvons constater, c'est que la position déterminée par le sexe, mortelle pour la femme, semble perdre son impact sur le personnage féminin, car Elisabeth Matrei s'invente, comme nous allons le voir plus loin, une généalogie dont elle exclut la différence sexuelle et où elle opte pour une généalogie "masculine » en s'identifiant à des hommes. Dans Malina, la différence sexuelle intervenait uniquement pour la fille et non pas pour le père qui pouvait prendre le rôle de la mère, dans Drei Wege zum see elle semble être levée, d'un point de vue symbolique, pour la fille également.

21 L'invention d'une généalogie qui ne prend pas en compte la différence sexuelle implique de lourdes conséquences. Pour quelles raisons? D’après le philosophe Pierre Legendre, à la base de toute vie communautaire se trouve nécessairement une référence absolue. De telles références donnent naissance, comme il l'explique, à des systèmes généalogiques, qui eux, servent de légitimation au pouvoir en vigueur. La référence absolue n'est pas vérifiable, car elle renvoie toujours au mythe fondateur, à ce qu'elle est elle-même. Selon Legendre, ce mythe se présente toujours sous forme d'image :

Nous sommes plantés là, comme des objets en représentation. Nous existons premièrement en tant que figurants, emblèmes, objets référés et fondateurs de références ${ }^{12}$.

$\mathrm{Au}$ lieu de forger une image unique, le roman Malina, conçu comme référence absolue sur un plan littéraire, introduit une suite d'images qui nous renvoient à l'anéantissement de la «fille», le personnage qui tente d'échapper à la violence masculine, et finalement au père.

Dans ce système, la figure de la fille n'a nul moyen de conquérir une place qui lui soit propre. La seule issue pour MOI serait le meurtre de l'assassin, qu'il s'agisse de la mère ou du père. Elle n'y procède pas («Ich kann ihn nicht töten» W3, 305). Tout au 
contraire, c'est elle qui sera assassinée («Es war Mord»W3, 336), victime peut-être consentante.

Nul ne s'immisce dans ce duel ${ }^{13}$. Cet aspect est accentué par une conception très particulière de la « Loi du Père ", c'est-à-dire du principe qui organise la mise en scène et la justification du pouvoir tant à l'échelle de l'individu qu'à l'échelle sociale ${ }^{14}$. Cette conception et la nature du conflit se conditionnent mutuellement. Bachmann élimine non seulement la différence sexuelle mais également le fait que la loi autorise la pérennité de l'espèce en laissant les enfants prendre la place des parents. Un exemple frappant : le père dans Malina ne respecte pas le tabou de l'inceste et il n'est pas soumis à la différence sexuelle mère-père. Il est l'instance qui tue, et qui exige une soumission inconditionnelle. Cette vision en quelque sorte abstraite de la Loi intervient déjà bien avant Malina.

Paru en 1961 dans le recueil Das dreißigste Jahr, le récit Unter Mördern und Irren, histoire d'une rencontre entre hommes, pose le même problème sous un angle bien différent. Ici apparaît un homme qui se désigne lui-même comme destiné à devenir un assassin. Curieusement, la guerre l'empêche d'accomplir son destin : il s'agit, pour lui, de tuer des hommes, non leurs substituts (des Américains, des Polonais). Même un séjour dans une clinique psychiatrique ne lui est d'aucun secours, car il en sort "guéri ", c'est-àdire « civilisé » : dès lors, il lui est impossible de tirer ni sur une «Abstraktion » ni sur un être humain. Par la suite, cet ex-assassin devient lui-même victime, tué par les participants à une rencontre d'anciens combattants qui se sentent provoqués par lui.

Dans le recueil, ce récit est placé avant Ein Schritt nach Gomorrha, où une femme songe à prendre une position masculine vis-à-vis d'une autre femme. C'est en quelque sorte l'histoire d'une offrande à la loi paternelle qui demande la séparation de ce qui n'a pas été séparé à l'origine : en l'occurrence l'abstrait et le concret. Cette offrande peut se faire indépendamment du sexe de la victime. L'important, c'est la position occupée dans une structure binaire: du côté de la loi (ce qui peut impliquer le meurtre légal); du côté de l'Autre (de cette loi, défini comme féminin par une longue tradition). La loi est conçue comme pure fonction violatrice et scindante, insensée, sans autre but que son autoreproduction, un aspect que Pierre Legendre appelle l'« instance du pouvoir à l'état pur, l'instance du pouvoir vide de contenu $»^{15}$. La "Loi du Père » a certes cette fonction, mais elle a en même temps un sens: celui de rendre possible une communication (au-delà de la symbiose mère-enfant préœdipienne), d'ériger un ordre symbolique et d'inscrire tout être humain dans l'espèce humaine, comme l'exprime encore Pierre Legendre :

La loi de l'espèce, dirais-je, fait Loi pour le sujet, ce qu'exprimaient très bien les glossateurs : la conjonction entre mâle et femelle conserve l'immortalité du genre humain ${ }^{16}$. Cette fonction ne semble pas entrer en jeu chez Ingeborg Bachmann.

L'intrigue du fragment Gier peut se lire comme une mise en scène encore plus radicale du fonctionnement de 1'« instance du pouvoir vide de contenu »: Elisabeth Mihailovics et le jeune employé forestier Slovène de son mari transgressent, chacun pour soi, une loi que l'autre ignore totalement. Cet acte est pour elle «mehr als jedes andere Gefühl, das Elisabeth je gespürt hatte und es gab nichts darüber hinaus » $(G, 52)$. Le résultat de ce comportement se présente par la suite comme aussi contingent et impératif que l'acte même: un double meurtre et un suicide, exécutés par le mari d'Elisabeth, représentant de la loi pour les deux personnages. La transgression mène donc droit à 
l'anéantissement. « Und so vergehen die Menschen, die [von Zufall] zu Zufall stolpernd, plötzlich unter ein nicht vorhandenes Gesetz [...]» $(G, 56)$. appartiennent à une même lignée, à une même nation qui cependant n'existe plus et
dont le père symbolique, l'empereur François Joseph, a commis une faute grave :

Sie kam auch, wie über so vieles über den See hinweg, sie nahm das Dreiländereck ins Aug, dort drüben hätte sie gern gelebt, in einer Einöde an der Grenze, wo es noch Bauern und Jäger gab, und sie dachte unwillkürlich, daß sie auch so angefangen hätte : Anmeine Völker ${ }^{17}$ Aber sie hätte sie nicht in den Tod geschickt und nicht diese Trennungen herbeigeführt, da sie doch gut miteinander gelebt hatten, immer natürlich in einem Mißverständnis, in Haßund Rebellion, aber man 
konnte ja von den Menschen wirklich nicht verlangen, daß sie sich von der Vernunft regieren ließen... (W2,444sq) des ancêtres après y avoir placé son père. Ainsi, elle cesse d'exister en tant qu'individu. L'harmonie imaginée par Elisabeth Matrei mène donc vers la mort symbolique du sujet féminin et de son désir érotique : premièrement, nous ne trouvons dans cette galerie aucune femme (c'est-à-dire que même la place de la mère a été symboliquement occupée par un homme) et deuxièmement, ce n'est que la mort du désir de l'« Autre » masculin qui permet l'identification avec lui, une identification par laquelle Elisabeth conquiert enfin une position dans un ordre apparemment neutre, mais en vérité «masculin».

\section{NOTES}

1. Voir, à ce propos l'édition critique de Todesarten (Ingeborg Bachmann, [Todesarten]-Projekt. Bearbeitet von Monika Albrecht und Dirk Göttsche. Piper, München 1995. Abrégé par la suite en $\mathrm{TP}$, suivi de l'indication du tome et de la page).

2. Il sera fait référence dans la suite de l'article aux Euvres complètes publiées par Christine Koschel, Inge von Weidenbaum, Clemens Münster. Piper, München 1978. Abrégé par la suite en : $\mathrm{W}$, suivi de l'indication du tome et de la page.

3. Nous pensons également au personnage de Jennifer dans la pièce radiophonique Ein Geschäft mit Träumen (Wl).

4. Cf., à propos de la notion d'amour, l'analyse de Undine geht dans Petervon Matt. Liebesverrat : Die Treulosen in der Literatur. Hanser, München 1989, p. 229-268.

5. Pour une analyse de ce texte cf. Kurt Bartsch. Ein Ort flir Zufälle. Bachmanns Büchnerpreisrede, als poetischer Text gelesen. In : Modern Austrian Literature. Special Ingeborg Bachmann Issue, vol. 18, n³/4, 1985, p. 135-145. 
6. Le thème de l'amour incestueux entre frère et sœur est déjà présent dans sa poésie, entre autres dans Das Spiel ist aus (Wl, 82sq) et dans le neuvième poème de Von einem Land, einem Fluß und den Seen, Wl, 92 sq.).

7. Nous avons entrepris une analyse exhaustive de cette "somme», en la comparant avec la prose de Marguerite Duras, dans le troisième chapitre de notre thèse de doctorat: La constitution du sujet chez Ingeborg Bachmann et Marguerite Duras. Paris III, 1994.

8. «... dieses Buch [Malina] ist für mich ein in sich geschlossener Anfang oder eine Ouvertüre... für dieses noch nicht geschriebene Buch [Todesarten]... »Ingeborg Bachmann : Wir müssen wahre Sätze finden. Gespräche und Interviews. Herausgegeben von Christine Koschel und Inge von Weidenbaum. Piper, München 1983, p. 95.

9. Mama muß damals in einer furchtbaren Verfassung gewesen sein, denn praktisch kämpften wir ja um dieses Kind, und Robert, der natürlich keine Ahnung haben konnte, auf welche Weise er zu zwei Müttern gekommen war, brachte Mama auch noch zur Verzweiflung... (W2, 443).

10. Ingeborg Bachmann. Gier (Fragment). Aus dem literarischen Nachlaß herausgegeben von Robert Pichl. In : Der dunkle Schatten, dem ich schon seit langem folge. Hg. Hans Höller. Locker, Wien 1982, p. 17-61.

11. ... Mama konnte nicht verstehen, daß eine Sechzehnjährige, der sie eben schon dreimal gesagt hatte, was man einem Mädchen eben zu sagen hat, sie plötzlich anschrie und fragte, ob denn Robert überhaupt ihr Kind sei, er könne nämlich genauso gut ihres, Elisabeths Kind, sein (W2, 443).

12. Pierre Legendre: L'Inestimable objet de la transmission. Étude sur le principe généalogique en Occident, Fayard, Paris 1985, p. 23.

13. La mère qui surgit dans les rêves de MOI est trop faible et Malina, conçu comme instance salvatrice au niveau de la narration, est un personnage neutre qui n'intervient pas dans les rêves mais seulement après.

14. Pour cette notion, cf. Legendre, op. cit., en particulier p. 43-50, 59-68, 312-314.

15. Ibidem, p. 44.

16. Ibidem, p. 45. Voir dans ce contexte également Der gute Gott von Manhattan où Ingeborg Bachmann attribue encore au bon Dieu cette fonction.

17. An Meine Völker, À Mes peuples : ainsi commençait le manifeste que l'empereur FrançoisJoseph fit afficher dans les villes et villages d'Autriche-Hongrie au moment de la mobilisation générale.

\section{RÉSUMÉS}

La prose tardive d'Ingeborg Bachmann est marquée, nous le savons, par une crise profonde des relations entre hommes et femmes. Crise qui s'exprime non seulement dans les conflits que vivent les protagonistes, mais aussi au niveau de la narration, par exemple à travers le maniement des perspectives narratives, pour la plupart du temps " masculines ", qui s'opposent à une perspective "de femme». Cet article a pour but de démontrer dans quelle mesure une impossible identification "féminine ", un effacement total de toute généalogie "féminine ", est responsable de cette crise qui aboutira à une neutralisation imaginaire de la différence sexuelle (à l'œuvre entre autres dans Malina et dans le récit Drei Wege zum See). 
Ingeborg Bachmanns späte Prosa ist von einer profunden Krise der Beziehungen zwischen Männern und Frauen gekennzeichnet. Einer Krise, die nicht nur in den Konflikten zum Ausdruck kommt, welche die Protagonisten mit einander austragen, sondern auch auf der narrativen Ebene, z.B. in der Handhabung der in der Regel «männlichen » Erzähl Perspektiven, die aber wiederholt mit einer «weiblichen» Perspektive konfrontiert werden. In diesem Artikel soll gezeigt werden, welche Rolle eine unmögliche "weibliche" Identifikation und das Auslöschen einer "weiblichen" Genealogie in dieser Krise spielen, die schließlich in eine imaginäre Neutralisierung der sexuellen Differenz mündet (welche unter anderem in Malina und in der Erzählung Drei Wege zum See sichtbar wird). 